\title{
Fragile X: Translation in Action
}

\author{
Mark F Bear ${ }^{\star,}$, Gül Dölen ${ }^{1}$, Emily Osterweil ${ }^{1}$ and Naveen Nagarajan ${ }^{1}$ \\ ${ }^{1}$ Department of Brain and Cognitive Sciences, Howard Hughes Medical Institute, Picower Institute for Learning and Memory, \\ Massachusetts Institute of Technology, Cambridge, MA, USA
}

Fragile $X$ is a synapsopathy - a disorder of synaptic function and plasticity. Recent studies using mouse models of the disease suggest that the critical defect is altered regulation of synaptic protein synthesis. Various strategies to restore balanced synaptic protein synthesis have been remarkably successful in correcting widely varied mutant phenotypes in mice. Insights gained by the study of synaptic plasticity in animal models of fragile $X$ have suggested novel therapeutic approaches, not only for human fragile $X$ but also for autism and mental retardation of unknown etiology.

Neuropsychopharmacology Reviews (2008) 33, 84-87; doi:10.1038/sj.npp.1301610; published online 17 October 2007

Keywords: autism; fragile x; metabotropic glutamate receptor; cerebral protein synthesis; synaptic plasticity; developmental disorder

\section{INTRODUCTION}

To state the obvious, proper brain function requires precise connections between neurons. The course connectivity of the brain is established before birth according to a rich interplay of genetic programs and intercellular signals. Synaptogenesis occurs even in the absence of functional synaptic transmission or electrical activity (Verhage et al, 2000). During early postnatal life, however, these connections are validated and refined under the influence of sensory experience. Although the fractional role in brain development is small, the quality of sensory experience from birth to adolescence is an essential determinant of adult brain capabilities and limitations (Katz and Shatz, 1996). This epoch is also noteworthy for the emergence of a large number of developmental brain disorders, ranging from autism to schizophrenia that might be considered 'synapsopathies' - diseases of synaptic development and plasticity. Clearly, the key to understanding these diseases is to explore how genes and environment interact to refine synaptic connections during postnatal life.

A popular model system to explore the interaction of genes and experience is the visual cortex. Although visual cortex is certainly not a structure where one expects to find the roots of psychiatric illness, the principles revealed here may be broadly applicable to synaptic development in more complex circuits devoted to thought, language, and emotion. Since the

${ }^{*}$ Correspondence: Dr MF Bear, Department of Brain and Cognitive Sciences, Howard Hughes Medical Institute, Picower Institute for Learning and Memory, Massachusetts Institute of Technology, 77 Massachusetts Avenue, Cambridge, MA 02139, USA, Tel: +161732 7002, Fax: +1 617324 7007, E-mail: mbear@mit.edu

Received 1 October 2007; accepted 1 October 2007 seminal studies of Hubel and Wiesel in the early 1960s, countless experiments have revealed the critical role of visual experience in establishing, refining, and maintaining the connectivity in visual cortex that is required for normal vision. Although other paradigms have been introduced over the years, the visual cortex offers the advantages that (1) the locus of change is known to be the cortex, not structures early in the visual pathway, (2) it is simple to manipulate visual experience, for example, by closing an eyelid, (3) simple manipulations have profound effects on cortical physiology that can be easily measured, and (4) changes in cortical physiology have clear behavioral consequences, that is, on vision. Research on visual cortical plasticity over the past 45 years has shown that multiple synaptic mechanisms contribute, including those of long-term potentiation (LTP), long-term depression (LTD), and metaplasticity (regulation of the properties of synaptic plasticity by the recent history of cellular or synaptic activity). Global regulation or gating of plasticity is also known to be provided by inhibition, neuromodulators, and the mechanisms of activity-dependent protein synthesis (reviewed by Bear, 2003). The recent introduction of mice as the species of choice for studies of visual cortical plasticity has not only enabled researchers to use genetic manipulations to bridge levels of analysis (eg from molecular mechanism to behavioral consequence) (Hubener, 2003), it has also opened the door to exploiting this extremely powerful model system to understand the interaction of genes and environment in the context of developmental brain disorders. In this brief review, we summarize recent work that suggests how the mutation responsible for fragile $\mathrm{X}$ syndrome (FXS), the most common inherited cause of human mental retardation and autism, disrupts synaptic plasticity and how it might be corrected with neuropsychopharmacology. 


\section{CORTICAL PLASTICITY IN THE MOUSE MODEL OF FXS}

Human FXS is caused by mutations in the FMR1 gene that encodes the fragile $\mathrm{X}$ mental retardation protein (FMRP). The large majority of cases of FXS are a result of transcriptional silencing of this gene. To facilitate studies of the pathophysiology of FXS, a mutant mouse has been created that lacks expression of the homologous gene Fmr1 (Consortium, 1994). We therefore began our study by examining the visual cortex of the Fmr 1 knockout (KO) mouse.

A standard method of measuring the performance of visual cortex in animals as well as humans is the visually evoked potential (VEP). VEPs reflect summed synaptic currents in the cortex and can be used to estimate both visual contrast sensitivity and acuity. We have used an awake mouse preparation in which VEPs are recorded from electrodes chronically implanted in layer 4 (the input layer) of primary visual cortex (Frenkel and Bear, 2004). VEP recordings from wild-type (WT) and KO mice at postnatal day 28 revealed no differences in visual responsiveness, suggesting that vision is grossly normal in the adolescent mice (consistent with human studies). However, the challenge of brief monocular deprivation (MD) revealed an interesting difference in visual cortical plasticity.

In WT mice, MD sets in motion a highly stereotyped series of changes characterized by the rapid loss of responsiveness of the deprived eye, followed by a compensatory increase in responsiveness of the non-deprived eye. Our studies in the KO mice suggested, surprisingly, that in the absence of FMRP, there is an accelerated response to deprivation. Such a phenotype could be explained in many ways, but clues come from the facts that (1) visual cortical plasticity depends on the rate of cerebral protein synthesis (Taha and Stryker, 2002) and (2) FMRP normally functions as a repressor of mRNA translation at synapses (Vanderklish and Edelman, 2005). Thus, a reasonable hypothesis is that in the absence of FMRP, excessive protein synthesis leads to hyperplasticity in visual cortex.

A widespread increase in the rate of basal protein synthesis has been reported in KO mice, using both direct measurement of incorporation of radioactive precursors into protein in vivo (Qin et al, 2005) and indirect estimates based on mRNA granule peaks on sucrose gradients (Aschrafi et al, 2005). This increased ongoing protein synthesis (and turnover) may account for another phenotype that has been observed in visual cortex, the increased density (by about 20\%), and length of dendritic spines (McKinney et al, 2005).

Dendritic spines are postsynaptic to excitatory glutamatergic synapses (reviewed by (Wallace and Bear, 2004). Thus, the finding of increased spine density could be interpreted as an indication of hyperconnectivity in fragile $\mathrm{X}$. Consistent with this interpretation, our own studies of synaptic development in primary hippocampal cultures have revealed an approximately $20 \%$ increase in the density of glutamatergic synapses in KO neurons (Nagarajan and Bear, unpublished). More work will be required to reconcile the findings that KO mice have WT levels of visual responsiveness despite increased excitatory synapse density; however, it is known that long thin spines harbor weak synapses. Thus, the increased density may compensate for the fact that the synapses are, on average, weaker and apparently more plastic in fragile $\mathrm{X}$.

\section{RESTORING NORMAL FUNCTION IN FRAGILE X BY REDUCING SIGNALING VIA METABOTROPIC GLUTAMATE RECEPTOR 5}

Even 'basal' protein synthesis in the brain is a function of the level of ongoing electrical and synaptic activity, begging the question of how synaptic tone regulates the level of protein synthesis. A few neurotransmitter and neurotrophin receptors have been implicated in local regulation of synaptic mRNA translation, but one that has come to the forefront is metabotropic glutamate receptor 5 (mGluR5), a metabotropic glutamate receptor. It is now well established that mGluR5 activation stimulates protein synthesis, and that many of the lasting consequences of mGluR5 activation require translation of preexisting dendritic mRNA (Grossman et al, 2006). A case in point is a form of LTD triggered by mGluR 5 activation in the hippocampus, which requires mRNA translation but not transcription (Huber et al, 2000). The same can be said of mGluR-dependent epileptogenesis (Merlin et al, 1998), dendritic spine elongation (Vanderklish and Edelman, 2002), LTP priming (Raymond et al, 2000), and LTP reversal (Zho et al, 2002) in the hippocampus.

An intriguing idea is that mGluR5 serves as a sensor of ongoing synaptic excitation in the brain and, among other actions, stimulates local mRNA translation so that the supply of rapidly turned over protein keeps up with demand. As with all biochemical pathways, this process is balanced by negative regulators. In the case of mGluR5dependent protein synthesis, one of these negative regulators might be FMRP. In the absence of FMRP, one might expect that the protein synthesis-dependent consequences of mGluR5 activation would be exaggerated - and indeed this is the case (Huber et al, 2002; Chuang et al, 2005).

Taken together, the findings that (1) mGluR5 stimulates protein synthesis and (2) FMRP negatively regulates protein synthesis led to the 'mGluR theory of fragile X,' which posits that the neurological and psychiatric symptoms of FXS are a consequence of exaggerated responses to mGluR activation (Bear et al, 2004). If this theory is valid, then it might be possible to correct aspects of fragile $\mathrm{X}$ by reducing signaling via mGluR5.

An exciting therapeutic implication of this theory is that drugs targeting mGluR5 might be therapeutically useful- possibly disease modifying. Consistent with this possibility, we found that the increased synaptic density of hippocampal neurons cultured from KO mice could be corrected by chronic blockade of mGluR5 using the selective antagonist MPEP (2-methyl-6-(phenylethynyl)pyridine; $2 \mu \mathrm{M}$ ) (Nagarajan and Bear, unpublished). Others have recently provided evidence that MPEP administered to KO mice in vivo can correct altered cerebral protein synthesis (Aschrafi et al, 2005) and audiogenic seizures (Yan et al, 2004). Ongoing experiments suggest that additional, widely varied KO mutant phenotypes can be corrected by a $50 \%$ reduction of mGluR 5 expression (Dolen et al, 2006). Thus, there is an increasingly strong case to be 
made for human clinical trials with mGluR5 antagonists for the treatment of human FXS (Bear, 2005).

\section{SYNAPTIC PROTEIN SYNTHESIS AND BRAIN FUNCTION}

It has been appreciated for many years that neuronal protein synthesis is a critical step in converting labile synaptic modifications into permanent changes that can store information. Regulation appears to occur both at the transcriptional and translational levels. Transcription appears to be influenced both by the integrated electrical activity of the neuron and rather globally by diffusely projecting neuromodulatory systems that can regulate the strength of memory storage depending on behavioral state and arousal. Translational regulation appears to be much more local and is closely tied to the recent history of activity at individual synapses. These insights have suggested ways that memory consolidation might be strengthened, for example in diseases such as Alzheimer's, by augmenting the actions of neuromodulators or specific transcription factors (Bear, 1997). Although few would argue that boosting protein synthesis can be beneficial for synaptic plasticity under some circumstances, recent work, including that on FXS, suggests that it is a double-edged sword.

With the sequencing of the human genome, the genetic basis for a number of developmental brain disorders has been revealed. Curiously, several single-gene disorders that are characterized by mental retardation and autism have been linked to a pathway that leads to excessive neuronal protein synthesis and expression. Besides fragile $\mathrm{X}$, these include tuberous sclerosis, Cowden syndrome, Angelman syndrome, Rett syndrome, and neurofibromatosis type 1 (reviewed by Kelleher and Bear, 2007).

We imagine that there is likely an inverted U-shaped function that relates neuronal network performance with synaptic protein synthesis (Figure 1). 'Neuronal network performance' obviously comprises a number of different domains that will have different behavioral read-outs. Thus, at any one level of synaptic protein synthesis, some functions might show substantial impairments, whereas others might be normal or even exceptional, possibly explaining restricted mnemonic savant abilities (a reflection of hyperplasticity) in some affected individuals.

If this conjecture is correct, drug treatments that affect synaptic protein synthesis will very likely have different effects in different patient populations. For example, an mGluR5 antagonist may very well cause cognitive impairment in normal humans, but restores cognitive functions in those with mental retardation and autism caused by excessive protein synthesis. These considerations will need to be taken into account in the design of clinical trials.

The postnatal emergence of cognitive impairment and autistic behavior likely reflect both the altered trajectory of brain development with excessive cerebral protein synthesis, and the ongoing manifestations of altered synaptic signaling. Although the ongoing excess in synaptic protein synthesis might be corrected at any age with appropriate therapy, the derailment in brain development might be difficult to reverse retrospectively. Thus, another issue that impacts clinical trial design will be age, as we would expect

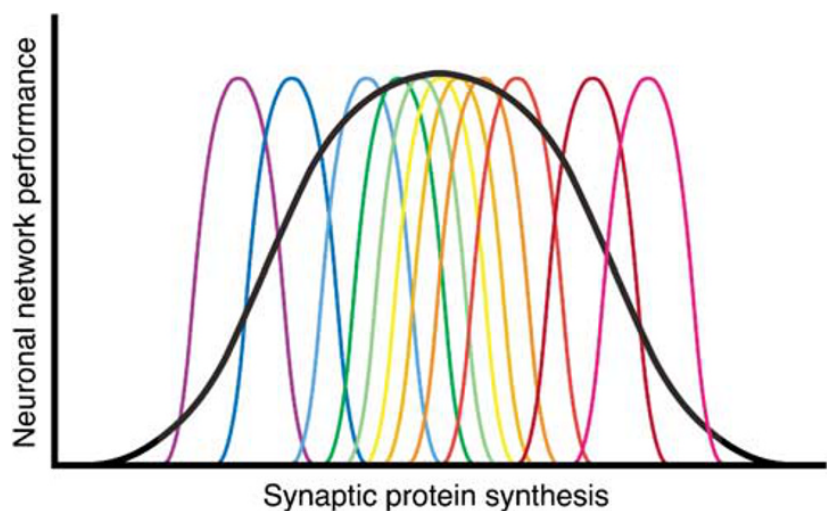

Figure I Hypothetical relationship of neuronal network function and levels of synaptic protein synthesis. In FXS, there is evidence that synaptic protein synthesis exceeds the optimum level and therefore causes an impairment in neuronal (and cognitive) function. This excessive protein synthesis can be corrected by reducing signaling via mGluR5. Other developmental disorders characterized by autism and mental retardation may similarly be associated with increased protein synthesis or decreased turnover. Note that 'neuronal network function' (thick black curve) represents some average of many separate domains (colored curves) Increased protein synthesis may cause a generalized impairment in many domains, while actually augmenting some, possibly leading to restricted savant abilities in affected individuals.

a priori the best outcome with early intervention. However, based on several animal studies (Aschrafi et al, 2005; McBride et al, 2005; Yan et al, 2005), we remain optimistic that significant benefit could be observed in some functional domains even if treatments were begun in adults.

Although not yet approved as human therapeutics, mGluR5 antagonists are currently entering into clinical trials for a broad range of psychiatric indications, including FXS. We are encouraged to believe that the recent progress in understanding the pathophysiology of fragile $\mathrm{X}$ may soon fulfill the promise of translating knowledge from basic neuroscience into the next generation of therapeutics.

\section{ACKNOWLEDGEMENTS}

This article summarizes research supported by grants from the NIMH, NICHD, NEI, FRAXA, and the Simons Foundation.

\section{DISCLOSURE/CONFLICT OF INTEREST}

Mark Bear has a financial interest in Seaside Therapeutics from which he has received personal compensation within the past 3 years. All other authors have no conflicts to disclose. Except as noted, no other financial support or compensation has been received from any individual or corporate entity over the past 3 years for research or professional service, and there are no personal financial holdings that could be perceived as constituting a potential conflict of interest.

\section{REFERENCES}

Aschrafi A, Cunningham BA, Edelman GM, Vanderklish PW (2005). The fragile $X$ mental retardation protein and group I 
metabotropic glutamate receptors regulate levels of mRNA granules in brain. Proc Natl Acad Sci USA 102: 2180-2185.

Bear MF (1997). How do memories leave their mark? Nature 385: 481-482.

Bear MF (2003). Bidirectional synaptic plasticity: from theory to reality. Philos Trans $R$ Soc Lond B Biol Sci 358: 649-655.

Bear MF (2005). Therapeutic implications of the mGluR theory of fragile X mental retardation. Genes Brain Behav 4: 393-398.

Bear MF, Huber KM, Warren ST (2004). The mGluR theory of fragile X mental retardation. Trends Neurosci 27: 370-377.

Chuang SC, Zhao W, Bauchwitz R, Yan Q, Bianchi R, Wong RK (2005). Prolonged epileptiform discharges induced by altered group I metabotropic glutamate receptor-mediated synaptic responses in hippocampal slices of a fragile $\mathrm{X}$ mouse model. J Neurosci 25: 8048-8055.

Consortium TD-BFX (1994). Fmr1 knockout mice: a model to study fragile X mental retardation. Cell 78: 23-33.

Dolen G, Osterweil E, Bear MF (2006). Genetic interaction between Fmr1 and Grm5: a role for mGluR5 in the pathogenesis of fragile X syndrome. Neuroscience Abstracts 33: 196.193.

Frenkel MY, Bear MF (2004). How monocular deprivation shifts ocular dominance in visual cortex of young mice. Neuron 44: 917-923.

Grossman AW, Aldridge GM, Weiler IJ, Greenough WT (2006). Local protein synthesis and spine morphogenesis: Fragile $\mathrm{X}$ syndrome and beyond. J Neurosci 26: 7151-7155.

Hubener M (2003). Mouse visual cortex. Curr Opin Neurobiol 13: 413-420.

Huber KM, Gallagher SM, Warren ST, Bear MF (2002). Altered synaptic plasticity in a mouse model of fragile $\mathrm{X}$ mental retardation. Proc Natl Acad Sci USA 99: 7746-7750.

Huber KM, Kayser MS, Bear MF (2000). Role for rapid dendritic protein synthesis in hippocampal mGluR-dependent long-term depression. Science 288: 1254-1257.

Katz LC, Shatz CJ (1996). Synaptic activity and the construction of cortical circuits. Science 274: 1133-1138.

Kelleher RJ, Bear MF (2007). The autistic neuron. Cell (in press).

McBride SM, Choi CH, Wang Y, Liebelt D, Braunstein E, Ferreiro D et al (2005). Pharmacological rescue of synaptic plasticity, courtship behavior, and mushroom body defects in a Drosophila model of fragile X syndrome. Neuron 45: 753-764.
McKinney BC, Grossman AW, Elisseou NM, Greenough WT (2005). Dendritic spine abnormalities in the occipital cortex of C57BL/6 Fmr1 knockout mice. Am J Med Genet B Neuropsychiatr Genet 136: 98-102.

Merlin LR, Bergold PJ, Wong RK (1998). Requirement of protein synthesis for group I mGluR-mediated induction of epileptiform discharges. J Neurophysiol 80: 989-993.

Qin M, Kang J, Burlin TV, Jiang C, Smith CB (2005). Postadolescent changes in regional cerebral protein synthesis: an in vivo study in the FMR1 null mouse. J Neurosci 25: 5087-5095.

Raymond CR, Thompson VL, Tate WP, Abraham WC (2000). Metabotropic glutamate receptors trigger homosynaptic protein synthesis to prolong long-term potentiation. J Neurosci 20: 969-976.

Taha S, Stryker MP (2002). Rapid ocular dominance plasticity requires cortical but not geniculate protein synthesis. Neuron 34: 425-436.

Vanderklish PW, Edelman GM (2002). Dendritic spines elongate after stimulation of group 1 metabotropic glutamate receptors in cultured hippocampal neurons. Proc Natl Acad Sci USA 99: 1639-1644.

Vanderklish PW, Edelman GM (2005). Differential translation and fragile X syndrome. Genes Brain Behav 4: 360-384.

Verhage M, Maia AS, Plomp JJ, Brussaard AB, Heeroma JH, Vermeer $\mathrm{H}$ et al (2000). Synaptic assembly of the brain in the absence of neurotransmitter secretion. Science 287: 864-869.

Wallace W, Bear MF (2004). A morphological correlate of synaptic scaling in visual cortex. J Neurosci 24: 6928-6938.

Yan QJ, Asafo-Adjei PK, Arnold HM, Brown RE, Bauchwitz RP (2004). A phenotypic and molecular characterization of the fmr1-tm1Cgr fragile X mouse. Genes Brain Behav 3: 337-359.

Yan QJ, Rammal M, Tranfaglia M, Bauchwitz RP (2005). Suppression of two major Fragile $\mathrm{X}$ Syndrome mouse model phenotypes by the mGluR5 antagonist MPEP. Neuropharmacology 49: 1053-1066.

Zho WM, You JL, Huang CC, Hsu KS (2002). The group I metabotropic glutamate receptor agonist (S)-3,5-dihydroxyphenylglycine induces a novel form of depotentiation in the CA1 region of the hippocampus. J Neurosci 22: 8838-8849. 